PROCEEDINGS OF THE

AMERICAN MATHEMATICAL SOCIETY

Volume 126, Number 11, November 1998, Pages 3397-3400

S 0002-9939(98)04346-9

\title{
ON REDUCTIVE GROUP ACTIONS AND FIXED POINTS
}

\author{
MINHYONG KIM \\ (Communicated by Eric M. Friedlander)
}

\begin{abstract}
Among analytic actions of reductive groups on projective varieties, we characterize the algebraic ones by the existence of fixed points for oneparameter subgroups. This applies to the problem of lifting the action of a compact Lie group on a projective manifold to a line bundle.
\end{abstract}

Let $G$ be a connected reductive algebraic group over $C$, let $X$ be a non-singular complex projective variety, and let $m: G \times X \rightarrow X$ be an analytic map giving a group action of $G$ on $X$. If $m$ is algebraic, then every one-parameter subgroup of $G$ will obviously have a fixed point on $X$ (by the valuative criterion of properness, for example). The purpose of this note is to give a simple proof of the following converse, which seems not to have been pointed out in the literature:

Theorem. Suppose every one-parameter subgroup of $G$ has a fixed point on $X$. Then $m$ is algebraic.

It is obvious that the hypothesis of the theorem holds if: $G$ itself has a fixed point; a Borel subgroup has a fixed point; or a maximal torus has a fixed point.

One can easily formulate a corollary of the following sort, whose proof is immediate:

Corollary 1. If, for some $x \in X$ and a maximal torus $T \subset G$, the orbit map $T \rightarrow X, t \mapsto t \cdot x$ is algebraic, then the $G$-action is algebraic.

Corollary 2. Let a compact connected Lie group $K$ act on the projective manifold $X$ as a group of analytic transformations, and let $L$ be an analytic (and therefore algebraic) line bundle on $X$. If each $S^{1} \subset K$ has a fixed point, then some power of $L$ is linearizable; that is, there is a $K$-action on some $L^{\otimes n}$ which covers the action on $X$.

Results of this sort abound in the literature on geometric quantization [6]. The existence of a fixed point for the $S^{1}$-action is equivalent to the existence of a moment map, which we actually use in the proof. However, in geometric quantization, the lifting is constructed in an elaborate manner using a connection whose curvature is equal to the Kaehler form. This therefore restricts the lifting only to specific line bundles. Also, the assumptions in the usual construction include the existence of a moment map for the whole group. Of course, the primary interest in that case being that of quantization, one is motivated to just consider such line bundles and to use the connection to construct certain Lie algebra homomorphisms.

Received by the editors August 21, 1992 and, in revised form, February 5, 1997.

1991 Mathematics Subject Classification. Primary 22E10.

(C)1998 American Mathematical Society 
On the other hand, this paper arose from an effort to understand why a lifting exists at all, and to clearly state that the more basic fact of the mere existence of a lifting holds for any line bundle, under the usual conditions, and for rather basic reasons (from the algebraic geometric viewpoint).

Proof of Corollary 2. The $K$-action extends to an analytic action of the reductive complexification $K_{C}$. Also, we can arrange it so that given a maximal (compact) torus $T$ in $K$ together with a decomposition $T=\left(S^{1}\right)^{n}$, the complexification of each factor together form a maximal algebraic torus $T_{C}=\left(C^{*}\right)^{n}$ of $K_{C}$. Each factor $C^{*}$ has the same fixed points as the corresponding factor $S^{1}$ in $T$, since the complex span of the vector field generated by $S^{1}$ gives the infinitesimal action of its complexification. Thus, each of these $C^{*}$-actions is algebraic, and hence, the action of $T_{C}$ is algebraic (by an argument to be presented below in the proof of the Theorem). Borel's fixed point theorem then implies that $T_{C}$ has a fixed point. As noted above, this implies that the $K_{C}$-action is algebraic. Therefore, the induced action on the Picard variety of $X$ is trivial. Now use [8], Proposition 1.5.

An example where the hypothesis fails to hold is provided by the action of $C^{*}$ on the elliptic curve $C^{*} / q^{Z}$ (for some $q \in C^{*}$ of infinite order) by translations. One notes that as $z \rightarrow 0$ in $C^{*}, z \cdot x$ winds around the elliptic curve infinitely many times, for any point $x$. The action is genuinely analytic, because the quotient map $C^{*} \rightarrow C^{*} / q^{Z}$ is analytic.

Consider $C^{*}$ acting analytically on an analytic space $X$. The isotropy groups for this action are closed analytic subgroups of $C^{*}$; that is, they are either all of $C^{*}$, finite, or of the form (finite) $\times q^{Z}$ for some $q$ of infinite order. The orbits are either a fixed point, $\simeq C^{*}$, or an elliptic curve, correspondingly. However,

Lemma. Suppose $C^{*}$ acts analytically on a projective manifold $X$ and has at least one fixed point. Then for each $x \in X$, the orbit map

$$
C^{*} \rightarrow X, \quad z \mapsto z \cdot x
$$

extends to a map $P^{1} \rightarrow X$.

Proof. Equip $X$ with a Kaehler metric invariant under the action of $S^{1} \subset C^{*}$, and let $\omega$ be the associated Kaehler form. Denote by $J$ the complex structure on $X$ so that $\omega(\cdot, J \cdot)$ is the Kaehler metric.

According to [4], the $S^{1}$-action has a moment map: if $V$ is the vector field on $X$ generated by $d / d \theta \in \operatorname{Lie} S^{1}$, there exists a smooth function $f$ on $X$ such that $d f=\omega(V, \cdot)$. In fact, it is a Morse-Bott function [1]. But then, for any vector field $W, d f(W)=\omega(V, W)=\omega(J V, J W)$ so that $J V=\operatorname{grad} f$. Thus, the flows of $J V$ must converge. It is easily checked, using the fact that the action is analytic, that $J V$ is the vector field generated by $r d / d r \in \operatorname{Lie} C^{*}$. This implies that for any $x \in X, 0 \cdot x:=\lim _{r \rightarrow 0} r \cdot x$ and $\infty \cdot x:=\lim _{r \rightarrow \infty} r \cdot x\left(r \in R_{>0}^{*} \subset C^{*}\right)$ exist, and are stationary points for grad $f$. But then $J V$ and hence $V$ vanish at these points. Therefore, $0 \cdot x$ and $\infty \cdot x$ are fixed points for the $C^{*}$-action and $\lim _{z \rightarrow 0} z \cdot x=0 \cdot x$, $\lim _{z \rightarrow \infty} z \cdot x=\infty \cdot x\left(z \in C^{*}\right)$.

This allows us to construct a continuous extension $P^{1} \rightarrow X$ which is then automatically analytic (and hence, algebraic).

The lemma implies, in particular, that the Zariski closure of each non-trivial orbit is an embedded $P^{1}$. This is a crucial point in the 
Proof of the Theorem. For any compact complex manifold $X$, Aut $(X)$, the group of holomorphic automorphisms of $X$, has the structure of a complex Lie group ([2]) and an action $m: G \times X \rightarrow X$ determines an analytic homomorphism $\tilde{m}: G \rightarrow \operatorname{Aut}(X)$ such that $m$ factors as

$$
G \times X \stackrel{\tilde{m} \times 1}{\rightarrow} \operatorname{Aut}(X) \times X \rightarrow X,
$$

the second arrow being the canonical action. Since $G$ is connected, we actually have $\tilde{m}: G \rightarrow \operatorname{Aut}(X)_{0}$, the connected component of the identity in $\operatorname{Aut}(X)$. When $X$ is projective, $\operatorname{Aut}(X)_{0}$ has the structure of a connected algebraic group acting algebraically on $X([5])$. By Chevalley's theorem $([9])$, Aut $(X)_{0}$ fits into an exact sequence of algebraic groups

$$
0 \rightarrow H \rightarrow \operatorname{Aut}(X)_{0} \stackrel{\pi}{\rightarrow} A \rightarrow 0
$$

where $H$ is linear and $A$ is an abelian variety.

Now, for a one-parameter subgroup $C^{*} \subset G$, consider the Zariski closure $\overline{\tilde{m}\left(C^{*}\right)}$ of its image in $\operatorname{Aut}(X)_{0}$. It is a connected algebraic subgroup ([7], p. 173) and so is of pure dimension. Suppose it is of dimension $\geq 2$. Then its generic orbit would be of dimension $\geq 2$. (The easiest way to see this is to note that the action generates at least two algebraic vector fields which are generically linearly independent.) However, since $\operatorname{Aut}(X)_{0} \times X \rightarrow X$ is algebraic,

$$
\overline{\tilde{m}\left(C^{*}\right)} \cdot x \subset \overline{\tilde{m}\left(C^{*}\right) \cdot x} \simeq P^{1},
$$

unless $x$ is a fixed point. Therefore, we must have $\operatorname{dim} \overline{\tilde{m}\left(C^{*}\right)} \leq 1$. So $\overline{\tilde{m}\left(C^{*}\right)}$ is either trivial, $\simeq C^{*}, \simeq C$, or an elliptic curve. But the last case is impossible by the non-existence of elliptic curve orbits on $X$. In the remaining cases, $\pi\left(\overline{\tilde{m}\left(C^{*}\right)}\right)=0$ since the projection $\pi$ is an algebraic map. Thus $\pi \circ \tilde{m}\left(C^{*}\right)=0$ for every oneparameter subgroup of $G$. But then, since the one-parameter subgroups generate a Zariski-dense subset (that is, the set of semi-simple elements) of $G$, we get $\pi \circ$ $\tilde{m}(G)=0$. That is, we actually have a map $\tilde{m}: G \rightarrow H$ which induces the $G$-action. However, an analytic map from a reductive group to a linear algebraic group is automatically algebraic. By taking a faithful algebraic representation, it suffices to check this for linear representations. Algebraicity of analytic representations is well known for semi-simple groups ([3], 2.5) and tori (by complete reducibility). This implies the statement for groups of the form (semi-simple) $\times$ (torus) since a representation $r: G^{\prime} \times G^{\prime \prime} \rightarrow G L_{n}$ factors as

$$
G^{\prime} \times G^{\prime \prime} \stackrel{r^{\prime} \times r^{\prime \prime}}{\longrightarrow} G L_{n} \times G L_{n} \rightarrow G L_{n}
$$

where $r^{\prime}:=r\left|G^{\prime} \times 0, r^{\prime \prime}:=r\right| 0 \times G^{\prime \prime}$ and the last map is multiplication in $G L_{n}$. The case of (semi-simple) $\times($ torus $) /($ finite), i.e. reductive groups, is then immediate, since an analytic representation of such a group is a quotient map coming from an algebraic representation of a finite cover.

The referee has suggested the possibility of a more algebraic proof along the following lines:

The key point in the proof is to show that the image of the map from $G$ to the automorphism group of $X$ lies in the linear part. For this, it suffices, as pointed out above, to show it for the $C^{*}$ subgroups. Now, since the image (if non-trivial) must be either $C^{*}$ or an elliptic curve, it suffices to show that the image has a non-trivial linear representation. But a fixed-point $p$ of the action would give rise to a non-trivial representation on some finite-order jet space of the functions at $p$. 


\section{REFERENCES}

[1] M. F. Atiyah, Convexity and commuting Hamiltonians, Bull. Lond. Math. Soc. 14 (1981), 1-15. MR 83e:53037

[2] S. Bochner and D. Montgomery, Groups on analytic manifolds, Ann. Math. 45, no. 3 (1947), 659-669. MR 9:174f

[3] A. Borel, Linear representations of semi-simple groups, in Proc. Symp. Pure Math. 29 (1975), 421-440, Amer. Math. Soc., Providence, RI. MR 51:8271

[4] T. Frankel, Fixed points on Kaehler manifolds, Ann. of Math. 70 (1959), 1-8.

[5] A. Grothendieck, Techniques de construction et théorèmes d'existence en géométrie algébrique IV, Seminaire Bourbaki 221, (1960/61).

[6] V. Guillemin and S. Sternberg, Geometric quantization and multiplicities of group representations, Invent. Math. 67 (1982), 515-538. MR 83m:58040

[7] S. Lang, Hyperbolic and Diophantine analysis, Bull. Amer. Math. Soc. 14, no. 2, (1986), 159-205. MR 87h:32051

[8] D. Mumford and J. Fogarty, Geometric Invariant Theory, 2nd ed., Springer Verlag, BerlinHeidelberg-New York (1982). MR 86a:14006

[9] M. Rosenlicht, Some basic theorems on algebraic groups, Amer. J. Math. 78 (1956), 401-443. MR 18:514a

Department of Mathematics, University of Arizona, Tucson, Arizona 85721

E-mail address: kim@math.arizona.edu 